\title{
An updated view of geriatrics at age 70, no longer just identified as a chronic, humanist patient
}

\author{
Achenbaum WA* \\ Graduate College of Social Work, University of Houston and the Institute for Spirituality and Health, Houston, TX 77006, USA
}

Teams of health-care professionals now routinely deploy best practices to help Baby Boomers and other aging patients stay healthy. These caregivers rely on new technologies, promising wonder drugs, and preventive measures, as well as evidenced-based trials, experiences, and common sense. Older patients are discouraged from remaining supine under geriatric care. Responsive patients in this regimen have an important, collaborative role in weighing health-care remedies; they can, for instance, best assess localized and generalized pain. Patients' complaints not only indicate how they manage to cope with discomfort, but sometimes their malaise may signal medical errors in the course of treatment. Some geriatric groups invite a seasoned layperson to join a patient's geriatric team. That person's primary role is to facilitate conversations between specialists in white coats and patients (and their families) who seem too scared, anxious or distracted to respond to medical caregivers' therapeutic preferences.

Ironically, because they are not strictly bound by medical protocols, such lay conduits serve a liminal role by offering advice, questions, and observations. Insights acquired when they were patients themselves validate their disinterested, Janus-faced role. Furthermore, rooted in a position of liminality affords these insiders/outsiders the privilege of identifying gaps in what has and has not been said about various caring options available. Finally, having become familiar with group dynamics outside clinics and hospitals empowers them to interject insights and admonitions that might be lost or forgotten due to the pressure of time or complexity of salient factors presented before patients and healthcare professionals.

I like serving as a seasoned veteran. I am a U.S. historian of aging who has devoted his career to studying and interpreting the meanings and experiences of growing older in past times and in the present. The professional gaze has personal counterparts. Since early middle age I had to wrestle with a major chronic illness. Adapting to a different lifestyle stoked my empathy for those who, like it or not, attend to diabetes, knee replacements, and various addictions.

My place on a geriatric team goes beyond fostering conversations between patients and health-care professionals. I organize training seminars; creating or altering curricula justifying CEUs in medicine; nursing; social work; and occupational therapy, making materials more comprehensible and unfamiliar in their cultures. Some of my responsibilities seem pedestrian: I generate data and polish articles for peer review; I plug organizational arrangements: I review funding proposals and budgetary demands by inter-professional team members, a significant number of whom I suspect will leave geriatrics for more lucrative specialties or alternative careers.

Geriatric health-care professionals tackle a concatenation of late- life vicissitudes, striving to restore, or sustain successful aging. Yet frailty and diminishing capacities bend the trajectories of growing older. It often falls on me to acknowledge that vulnerabilities cast shadows over the life course. Palliative care rarely trumps terminal illness. Accordingly, I occasionally complement the work of registered nurses in effecting the transition from growing older to addressing life as dying unfolds. When medical interventions are exhausted, such existential moments seem predictable, while at other times the ravaging hour of death occurs unexpectedly, jarring our sense of ordinary time.

I was very flattered to be invited in 2011 to reflect on my medical and academic experiences in the Journal of Pain Symptom Management. There I published "This chronic patient becomes a humanistic patient who helps clinicians" six months after I turned 65. The piece in print resembled a lengthy editorial more than a scholarly contribution. After describing two early bouts with chronic illness-bipolarity at age 41 and prostate cancer in my 50s--I described how I framed these incidents in teaching nurses, residents, interns, and social workers. I embraced the patient's perspective, elaborating on how I wanted to be listened to and cared. I underscored the importance of civility and compassion in conversations between the care deliverer and patients often anxious about procedures and outcomes. And in the process of relating my story, I came to trust my own voice-one of being a compassionate, sentient human being-no longer content with being a passive bystander.

Now, as I approach my $70^{\text {th }}$ birthday, I have been invited to give an update. I am pleased to write about my richer appreciation of nurses delivering palliative care with honest words and consoling gestures. It turns out, however, that I have much to report that relates to my physical and mental resilience. I am semi-retired from an academic career to which I devoted enormous time and energy. The contours of my identities have changed with new opportunities. To find meaning in my vocation, I have moved to a research institute near Houston's Texas Medical Center, where I have more time to think and write than has been my lot since completing my dissertation.

On a personal note, I remarried, spiritually and happily. Then a dark face of aging appeared. My bride was diagnosed with anal cancer nine weeks after the wedding. Despite terrific care, she spent 71 days in the hospital. She survived, but radiation really damaged her middle.

Correspondence to: Achenbaum WA, Graduate College of Social Work, University of Houston and the Institute for Spirituality and Health, Houston, TX 77006, USA, E-mail: wachenba@Central.UH.EDU

Received: November 14, 2016; Accepted: December 12, 2016; Published: December 15, 2016 
The protracted recuperation made me an uncomfortable caregiver. I became silent when the oncologist and other team members entered the room; they paid attention only to my wife, often too disoriented to remember details that presented that day or day before. Even when I displayed an alpha-dog's anger, my questions were usually ignored or vaguely addressed.

My own life had to get on while worrying constantly about my wife's future. Cataract surgery was nonetheless warranted in the early stages of my wife's healing. The procedures went well, the results were excellent, but I detached a retina four months after my operation. The surgeon repairing the damage was convinced that I had delayed presenting myself so as to complicate his remedy. Unable to change his opinion, I tried to win him over--suggesting our time at the University of Michigan overlapped. The surgeon would have none of it. Still, he did a great job--good enough that I almost shrugged off his parting shot about not waiting so long next time I had to come to his office

My second acute accident proved more complicated. For more than a decade after my prostate surgery I had suffered urinary incontinence. Stress worsened during my wife's hospitalization. Only an artificial sphincter held hope for relieving the embarrassment of increasingly frequent accidents. Although the device was successfully implanted, my patient education was less felicitous. At times, I wondered if the integrity of the sphincter mattered more than my recovery. I did not realize the magnitude of the problem until I heard urologists discuss "renal failure" and suspend lithium-- consequences of holding several liters of urine over my bloated middle.

So, this has been the year in which I experience acute maladies interacting with chronic ailments. Scientific procedures transmogrify any course in miracles. In part my attitude towards fear and pain has changed. I remain an optimist: my body faces different demands than it did at 40 , but it remains functional enough to celebrate life with my wife, family, and friends. That said, I get less work done when doctors' appointments fill our weeks.

People seem to listen when I talk about life instead of history or gerontology. I realize that time is hardly meaningless to Type As, but conserving energy and maintaining relationships is always a timely priority. Hard knocks have made me slightly humbler. Exulting in what life fosters gratitude-even though I choose not to ignore the realities of suffering. I perceive deeper meanings in the human bonds that gird conservations and interventions in hospital floors. Armed with a deeper sense of death's immanence. I believe my fellow gerontologists should pay greater attention to the value of palliative care in assuaging pain and frailty over the life course. At 70 I spend much time reflecting on gerontology's connections to thanatology. Despite gains in individual and societal longevity, dreadful medical crises such as AIDS and ZIKA are not invariably postponed to my generation's Fourth Age; Boomers know that some of late life's mysteries precede that first Social Security check

And that leads me to one other major health events in 2016. In May, at home out of the blue, I had a stroke despite adhering to behavioral and pharmaceutical precautions. I was very lucky: immediate access to a remarkable stroke center worked in my favor; I suffered no side effects. I had enough cognition in the ambulance to dread uncertainties about paralysis, about labored speaking, and about whether I would die after a second stroke.

Spiritual readings and medication became at forty, after the bipolar diagnosis, a major part of my journey to healthfulness. Now, three decades later, spiritual practices cut deeper. Instead of plea bargaining with some Ultimate Reality, as if relying on a hot line to the Divine assigned to me in emergencies, I let go of control, I surrendered my conniving and negotiating. I am less cocksure at 70 than at 65 about facing the finality of finitude. Although no one could understand me, I stayed hopeful, more stoic than benign: I could not control whatever transpired.

The bio-medico-social-psychological model presented nearly 60 years ago, now embraces a spiritual dimension. Someday we need less voices like mine advocating for spirituality, but so far health care practitioners allocate little of their time or literature reviews in dialogic exchanges with patients or geriatric team members. This makes it more important to sensitize older patients to their own capacities for compassion, forgiveness, and love. Developing a spiritual reservoir early in the life course and surely in young old age may make it easier to face finitude and end-of-life decisions and care.

\section{References}

1. Achenbaum WA (2012) This chronic patient becomes a humanistic patient who helps clinicians. J Pain Symptom Manage 44(5):784-788. [Crosssref]

Copyright: (C2016 Achenbaum WA. This is an open-access article distributed under the terms of the Creative Commons Attribution License, which permits unrestricted use, distribution, and reproduction in any medium, provided the original author and source are credited. 